\title{
Remodeling beim Asthma
}

\section{Remodeling in Asthma, a Comprehensive Review}

A. Gillissen

Klinik für Lungen- und Bronchialmedizin, Klinikum Kassel

\section{Lernziel}

$\nabla$

In diesem Artikel wird beschrieben, was unter Remodeling verstanden wird und was dies für den Asthmapatienten bedeutet. Es wird veranschaulicht, welche Zellstrukturen dabei betroffen sind, ob diese Veränderungen irreversibel oder reversibel sind und inwieweit die aktuell auf dem Markt befindlichen Medikamente oder evtl. zukünftig zur Verfügung stehende Pharmaka diesen Prozess für den Patienten spürbar beeinflussen können.

\section{Einleitung \\ $\nabla$}

Der Begriff „Remodeling“ ist nicht einheitlich und allgemein verbindlich definiert. Ungeachtet dessen werden unter Remodeling strukturelle Veränderungen sowohl in den großen als auch in den kleinen Atemwegen verstanden, die im Rahmen der persistierenden bronchialen Entzündung beim Asthma entstehen und auch persistieren können. Remodelingprozesse wurden in allen Asthmagraden dokumentiert und betreffen den Verlust der epithelialen Integrität der Bronchialschleimhaut („shedding“ des Atemwegsepithels), Verdickung der subepithelialen Basalmembran, die subepitheliale Fibrosierung, die Drüsenzellhyperplasie, die Hypertrophie der Bronchialschleimhautmuskulatur, Chondropathie der bronchialen Knorpelspangen und die Hypervaskularisierung der Bronchialschleimhaut ( $\bullet$ Abb. 1) [1]

Der Begriff Remodeling beschreibt strukturelle Veränderungen in den Atemwegen.

Die mit zunehmendem Asthmaschweregrad und/ oder einer unzureichenden oder fehlenden inhalativen Kortikosteroidtherapie assoziierten Lungenfunktionsverschlechterung sowie die bronchiale Hyperreaktivität und die Asthmasymptome fußen letztendlich auf diesen pathophysiologischen Veränderungen. Aus diesen Erkenntnissen werden heute zunehmend neue Ansätze zu einer zielgerichteten Therapie entwickelt und mittlerweile auch klinisch getestet [2]. Als Faustregel gilt: Je länger ein Asthma besteht und je schlechter es antiinflammatorisch behandelt wird, desto ausgeprägter ist der bronchiale Remodelingprozess und desto schlechter das Therapieansprechen. Der Remodelingprozess wird somit als Folge einer unzureichend inhibierten bronchialen Entzündung verstanden. Bezüglich des Remodelings beim Asthma und des bei der chronisch-obstruktiven Lungenerkrankung (COPD) gibt es diverse Überlappungen [3,4].

In diesem Artikel wird ein Überblick über die verschiedenen Remodelingprozesse beim Asthma und die sich hieraus ableitenden Konsequenzen für die Asthmatherapie einschließlich eines Blickes in die Zukunft gegeben.

\section{Veränderung des Atemwegsepithels $\nabla$}

Das Atemwegsepithel bildet die Grenze zwischen der Umwelt und der Lunge. Epithelzellen haben aber nicht nur eine mechanische Funktion, sondern besitzen auch metabolische und immunologische Fähigkeiten, da sie proinflammatorische Zytokine, Wachstumsfaktoren und Mediatoren sezernieren und so am asthmatischen Entzündungsgeschehen partizipieren.

Beim Asthma werden im Bereich des Atemwegsepithels folgende Veränderungen beobachtet:

Das Atemwegsepithel hat mechanische, metabolische und immunologische Funktionen.

- Shedding (Abschilfern) des Atemwegsepithels [5]

- Verlust zilientragender Zellen,

- Goblet-Zellhyperplasie

- Vermehrte Freisetzung von Wachstumsfaktoren, wie z.B. TGF (transforming growth factor), PDGF (Platelet-derived growth factor), EGF (epidermal growth factor), b-FGF (basic fibroblast growth factor), IGF-1 (insulin-like growth factor-1), ET-1 (Endothelin-1) und HB-EGF (Heparin-binding epidermal growth factor),
VNR

2760512012137991405

Bibliografie

DOI http://dx.doi.org/ 10.1055/s-0032-1309753 Pneumologie 2012; 66:

345-355

(c) Georg Thieme Verlag KG Stuttgart · New York

ISSN 0934-8387

\section{Korrespondenzadresse}

Prof. Dr. A. Gillissen

Klinik für Lungen- und Bronchialmedizin Klinikum Kassel GmbH Möncheberg-Str. 41-43 34125 Kassel adrian.gillissen@klinikumkassel.de 


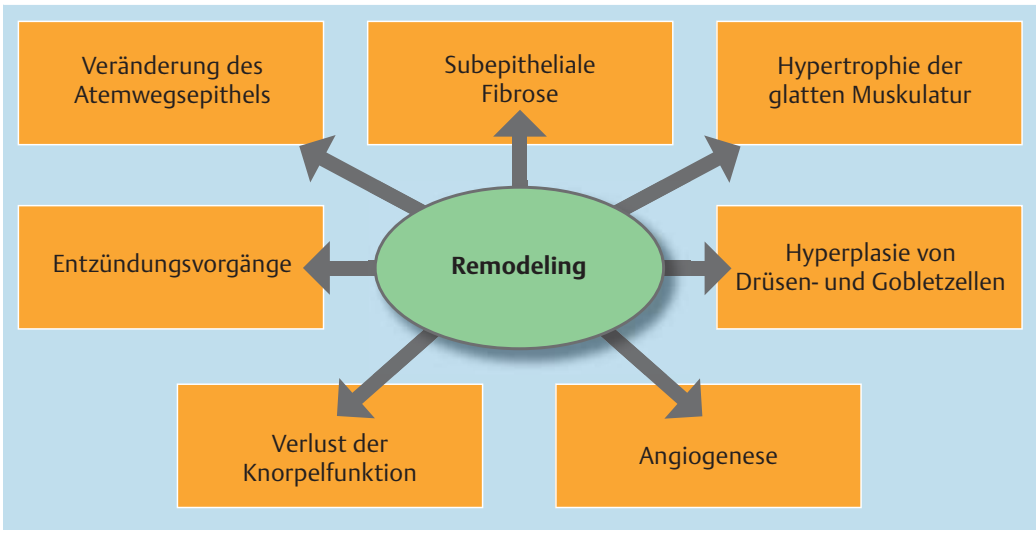

Abb. 1 „Remodeling“ ist kein einheitlich und allgemein verbindlich definierter Begriff. Ungeachtet dessen wird darunter die Summe einer komplexen Entzündung verstanden, die alle zellulären Strukturen der Atemwege umfasst [12].

die alle die Fibroblastenproliferation und die extrazelluläre Matrixproduktion im Rahmen des Remodelingprozesses stimulieren.

- Gesteigerte Expression von z.B. EGFR (epithelial growth factor receptor), dem Transskriptionsfaktor STAT-1, Amphiregulin und Muzin-Glykoproteinen (MUCs).

Dabei werden die Ursache und die pathophysiologische Bedeutung des Abschilferungsprozesses kontrovers diskutiert. Bei dem Shedding könnte es sich lediglich um ein Artefakt bei der bronchoskopischen Probenentnahme handeln, denn Epithelzellen lösen sich bei einer Entzündung ohnehin leicht von der Basalmembran oder der Zellumsatz ist bei Asthmatikern gegenüber Lungengesunden erhöht [6].

Das Ausmaß des Epithelschadens korreliert mit der bronchialen Hyperreaktivität und der klinischen Asthmaschwere. Die protektive Zellbarriere schützt im Normalfall die Basalmembran und die subepithelialen Strukturen, während ein Schaden die Empfindlichkeit gegenüber proinflammatorischen Triggern (z.B. Allergene, unspezifische Asthmaauslöser wie kalte Luft, Stäube) erhöht [7].

Die bronchiale Hyperreaktivität ist unmittelbare Folge des bronchialen Epithelschadens.

Der Mukus ist Teil der mukoziliären Clearance, da er Bakterien, eingeatmete Partikel und abgeschilferte Zellen bindet und das darunterliegende Bronchialepithel schützt. Die Mukusüberproduktion ist ein Charakteristikum der asthmatischen Entzündung und ist typisch für das unzureichend antiinflammatorisch behandelte Asthma. Hier sind das vermehrte Vorkommen von Goblet-Zellen und die Hyperplasie von submukösen Drüsenzellen typisch, die diese Mukusmengen produzieren. Da Epithelzellen EGFR sezernieren und dieses wiederum die MUCs-Expression steigert, sind diese Zellen in der Lage, die in ihr eingebetteten Drüsenzellen zu stimulieren und damit die Mukusproduktion zu steuern [8]. Andere Stimuli gehen von den Th2-Lymphozyten und den von ihnen sezernierten Zytokinen aus. IL (Interleukin)-13 bewirkt z.B. eine Überexpression von MUC5AC, welche wiederum eine Globlet-Zellmetaplasie und in der Folge eine Aktivierung chemotaktisch rekrutierter neutrophiler Granulozyten nach sich zieht [9].

Die Epithelzellen von Asthmatikern produzieren auch vermehrt STAT-6, einen Transkriptionsfaktor, der ebenso wie CLCA1 $\left(\mathrm{Ca}^{2+}\right.$-activated chloride channel-1) und Amphiregulin, einen epithelialen Wachstumsfaktor, der von stimulierten Mastzellen freigesetzt wird und die MUC-Expression im Atemwegsepithel steigert $[7,10]$.

\section{Extrazelluläre Matrix}

Unter der extrazellulären Matrix (ECM) werden die verbindenden und dynamischen Strukturen verstanden, die das mechanische Gerüst eines Zellverbandes bilden. Sie verbindet Zellen und garantiert den räumlichen Aufbau der Atemwege. Die Bronchialwände sind bei Patienten mit einem Asthma vor allem im subepithelialen Bereich und insbesondere unterhalb der Basalmembran verändert.

\section{Die extrazelluläre Matrix bildet das mechanische} Gerüst von Zellverbänden.

An Asthmapatienten gewonnene Biopsien der Bronchialschleimhaut zeigen eine typische Entität des Remodelings, nämlich die in der Lamina reticularis auftretende subepitheliale Fibrosierung. Diverse Studien konnten bei zum Tode führenden Asthmaanfällen, bei Kindern mit schwer zu behandelndem Asthma, aber auch bei leichteren Asthmaformen und selbst bei der atopischen Rhinitis eine Fibroblastenvermehrung und Fibrose in diesem Bereich nachweisen [1,11]. Die Fibrosierung entsteht aus einer vermehrten Deposition von extrazellulären Matrixproteinen einschließlich Kollagen I, III und V, Hyaluran, Versican, Biglycan sowie Fibronektin, Laminin $\alpha 2 / ß 2$, Tenaskin, Lumikan und Proteoglykan aktivierter Fibroblasten und vermindertem Kollagen IV, Elastin und Decorin [12]. Da die Lamina reticularis direkt unterhalb der Basalmembran liegt, führen diese Vorgänge zu einer mikroskopisch sichtbaren Membranverdickung, die wiederum ein Charakteristikum des nicht adäquat behandelten Asthmas ist.

Fibrosierungsvorgänge der Lamina reticularis bewirken eine Verdickung der Basalmembran.

Diese Veränderungen lassen sich sogar in vivo mit der fiberoptischen Konfokal-Fluoreszenzmikroskopie im Rahmen einer Bronchoskopie nachweisen [13]. Interessanterweise gibt es aber auch Hinweise, dass die vermehrte Verdickung der 
Bronchialwand zu einer Versteifung führt, die wiederum vor einer Bronchialobstruktion schützen kann [14]. Passend dazu zeigen Untersuchungen im allergeninduzierten Rattenmodell, dass die bronchiale Hyperreaktivität zwar mit zunehmender bronchialer Entzündung steigt, dass sie aber mit zunehmender Fibronektin- und Kollagendeposition wieder sinkt [15].

Die Proteoglykandeposition in der extrazellulären Matrix und die Proteoglykanfreisetzung von aktivierten Fibroblasten korrelieren mit dem Ausmaß der bronchialen Hyperreaktivität bei Asthmatikern [16]. Zudem fördert eine Proteasen-Antiproteasenimbalanz zugunsten der von interstitiellen Zellen, Makrophagen und neutrophilen Granulozyten freigesetzten Proteasen diese Fibrosierung. Dabei spielen vor allem die Matrix-Metalloproteinasen (MMPs), die bei Asthmapatienten vermehrt im Sputum oder in der bronchoalveolären Lavage nachgewiesen werden und dann invers mit der FEV1 korrelieren, eine wichtige Rolle [17]. Sie wirken chemotaktisch auf eosinophile Granulozyten, fördern den Matrixumbau, die Angiogenese und die Hypertrophie der glatten Muskulatur [17].

\section{Glatte Bronchialmuskulatur \\ $\nabla$}

Die glatten Muskelzellen in den Atemwegen sind die Effektorzellen der asthmatischen Entzündung, in dem sie die Muskelzellen vergrößern (= Hypertrophie) und an Zahl zunehmen (=Hyperplasie). Dabei können sie sich schneller kontrahieren und sie können sich weiter als im nicht aktivierten Zustand zusammenziehen, womit sie aktiver und effektiver sind als die von Gesunden.

Hypertrophie und Hyperplasie sind charakteristische Veränderungen der glatten Muskelzellen.

Diese Veränderungen sind durch die vermehrte Expression von sm-MLCK (smooth muscle myosin light chain kinase), Transgelin (sm-22) und smMHC (myosin heavy chain) erklärbar, wie Biopsiestudien bei Asthmatikern belegten [18]. Die erhöhte Expression kontraktiler Proteine korreliert mit der bronchialen Empfindlichkeit auf Methacholin. Gleichzeitig verlangsamt die Fähigkeit derart sensibilisierter glatter Muskelzellen, sich schnell wieder zur relaxieren [19]. Die asthmatische Entzündung bewirkt letztendlich eine vermehrte Muskelmasse in den Atemwegen. Die Muskelkraft ist dabei proportional zur Muskelmasse und bewirkt effektiv die Verengung der Atemwege bei einem Asthmapatienten. Hierauf beruht übrigens die Wirksamkeitshypothese der Thermoplastie (s.u.), da diese mittels lokaler Wärmeapplikation die Muskelmasse und somit auch die zur Verfügung stehende Kraft, die Atemwege zu kontrahieren, reduziert [20,21].
Aktivierte glatte Muskelzellen sezernieren proinflammatorische Mediatoren.

Glatte Muskelzellen können sich auch verändern, indem sie einen sekretorischen und proliferativen Phänotyp annehmen und sogar in das subepitheliale Kompartiment migrieren können [22]. Aktivierte Myozyten können proinflammatorische, proangiogene und Pro-Remodelingfaktoren, einschließlich Eotaxin, VEGF (vascular endothelial growth factor), CTGF (connective tissue growth factor) und antimitogene Faktoren wie PGE2 (Prostaglandin-E2), produzieren [23]. In-vitro-Zellkulturen belegen, dass glatte Muskelzellen von Asthmapatienten durch eine Veränderung der ECMProteindeposition, einer beschleunigten mitochondrialen Biogenese und Aktivität schneller proliferieren als die von Gesunden [24]. Diese Entzündungsmediatoren haben eine chemotaktische, zellproliferative und eine lebensverlängernde Wirkung auf Entzündungszellen, wie z.B. auch auf Mastzellen. Die Zell-Zell-Interaktion von glatten Muskelzellen und Mastzellen verstärkt die komplementinduzierte Mastzellendegranulation [25].

Die Muskelmasse der Atemwege korreliert nicht mit dem Patientenlebensalter.

Umgekehrt haben Mastzellen jedoch keinen Einfluss auf die Proliferationsfähigkeit und das Überleben der glatten Muskelzellen. Ungeklärt ist, warum es keine klare Beziehung vom Patientenalter und der Erkrankungsdauer zum Ausmaß der Muskelmasse gibt. So können z.B. schon junge Kinder eine ausgeprägte Verdickung der glatten Muskulatur in den Atemwegen aufweisen. Allerdings gibt es sehr wohl eine lineare Beziehung zur Asthmaschwere [23,26].

Remodeling kann auch durch mechanischen Stress, z.B. durch wiederholte Bronchokonstriktionen, d.h. unabhängig von einer entzündlichen und/oder allergischen Reaktion ausgelöst werden. Grainge et al. untersuchten Asthmapatienten, die sie bis zu viermal mit Metacholin oder einem Hausstaubmilbenextrakt inhalativ provozierten. Bronchoskopisch wurden vor und 4 Tage nach der Provokation Schleimhautbiopsien gewonnen. Nur in der Allergenprovokationsgruppe erhöhten sich die Eosinophilen, aber in beiden Gruppen kam es zu einer Vermehrung des subepithelialen Kollagens und der intraepithelialen Drüsenzellen.

Somit können auch wiederholte Bronchokonstriktionen, die durch unspezifische Reize ausgelöst wurden, ein Remodeling provozieren. [27]. Wie In-vitro-Studien zeigen, führt mechanischer Stress zu einer Stimulation der Atemwegsepithelzellen [28]. Diese wiederum setzen Entzündungsund Wachstumsmediatoren frei, die ihrerseits Myofibroblasten und Myozyten stimulieren und a) zur Proliferation und b) zur Freisetzung weite-

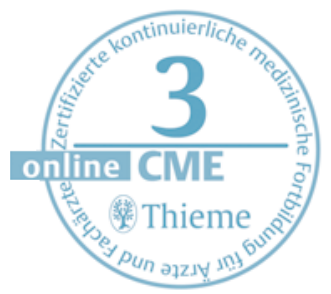


rer Mediatoren anregen. Damit stehen die auf einer immunologischen Reaktion basierenden Remodelingprozesse (z.B. allergische Entzündung) solchen gegenüber, die nur durch einen mechanischen Reiz hervorgerufen werden [29]. In praxi werden sicherlich beide Mechanismen gemeinsam auftreten.

Mechanischer Stress fördert in Myozyten und Myofibroblasten die Zellproliferation und Mediatorfreisetzung.

Glatte Muskelzellen sind interaktiv und kommunizieren mit den sie umgebenden Zellkompartimenten, nämlich dem Atemwegsepithel, dem parasympathischen Nervensystem und der ECM. Erkenntnisse zu dieser Zell-Zell-Interaktion haben in der Vergangenheit und werden in der Zukunft zu der Entwicklung von Medikamenten führen, die einen direkten oder indirekten Einfluss auf den Muskelton in den Atemwegen bewirken (s.u.).

- Atemwegsepithel: Das bei Asthmapatienten veränderte L-Arginin-Gleichgewicht bewirkt eine Senkung der NOS (nitric oxide synthase). In der Folge ist das zelluläre NO (Stickstoffmonoxid) erniedrigt und Peroxynitrit $\left(\mathrm{ONOO}^{-}\right)$ erhöht. Ferner kommt es beim Asthmatiker zu einer Steigerung der Muskelmasse der glatten Muskulatur. Den gleichen Effekt hat die beim Asthma gefundene erhöhte Arginaseaktivität mit einer Steigerung der Polyamine L-Prolin und L-Ornithin [30,31].

- Parasympathisches Nervensystem: Die Regulation der neuronalen Freisetzung von Acetylcholin aus den parasympathischen Nervenenden in entzündeten Atemwegsepithelien ist ein Charakteristikum der eosinophilen Entzündung, wobei Acetylcholin postjunktionale Effekte auf glatte Muskelzellen besitzt und diese in der Folge hypertrophieren. Acetylcholin, die im Rahmen der Entzündung freigesetzten Wachstumsfaktoren (z.B. EGF, PDGF) und Entzündungsmediatoren bewirken in diesem Rahmen eine Steigerung der muskelspezifischen Genexpression (sm-MLCK und sm-MHC) und Zellproliferation [32,33]. Möglicherweise spielt in diesem Zusammenhang auch nonneuronal freigesetztes Acetylcholin eine Rolle.

- Extrazelluläre Matrix: Die o.g. Veränderungen der ECM betreffen auch die glatte Muskulatur der Atemwege, da sie diese umgibt und bei der asthmatischen Entzündung deutlich vermehrt nachgewiesen wird. Dabei verhält sich die Elastinmenge invers zur bronchialen Hyperreaktivität [34]. Die Expression von ECM-Komponenten (s.o.) wird von profibrotischen Proteinen, wie TGF-ß (transforming growth factorbeta), CTGF (connective tissue growth factor) und VEGF (vascular endothelial growth factor) gesteigert. Die vermehrte Deposition von ECM-Proteinen steigert die Muskelfunktion einschließlich deren Proliferation und deren Kontraktilität. Wesentliche Trigger sind dabei Integrine und Laminine, die wieder über Wachstumsfaktoren, wie z.B. dem TGF-ß, stimuliert werden. Zudem regulieren sie eine Fülle weiterer, für die Muskelzellen wichtige Eigenschaften, wie deren Überlebenszeit, Migrationsverhalten, Zytokinsynthese und Zellreifung [35]. Muskelzellen und die ECM-Bestandteile beeinflussen sich dabei gegenseitig. Die Interaktion zwischen den glatten Muskelzellen der Atemwege und der ECM bildet einen wesentlichen Bestandteil des Remodelingprozesses der asthmatischen Entzündung, zumal die einmal aufgetretenen zellulären Veränderungen nicht mehr rückgängig zu machen sind, auch wenn die Entzündung abgeklungen ist.

\section{Therapiemöglichkeiten des Remodelings}

$\nabla$

Das primäre Ziel der Asthmatherapie ist nicht, Remodeling zu reduzieren, sondern die Symptome des Patienten zu lindern, Exazerbationen zu vermeiden und die Lungenfunktion zu verbessern. Da dem Asthma aber eine immunologisch gut charakterisierbare Entzündung in den Atemwegen zugrunde liegt, lassen sich mit deren therapeutischer Hemmung die besten Langzeiteffekte erzielen [36-38].

Die Verhinderung des Remodelings als therapeutisches Ziel alter und neuer Therapeutika.

Die Gruppe mit der stärksten und breitesten antiinflammatorischen Wirkung stellen die Kortikosteroide dar, obwohl Substanzen wie der Leukotrienrezeptorinhibitor Montelukast und der IgEAntikörper Omalizumab auch antientzündlich wirken und ebenfalls bestimmte Strukturveränderungen in den Atemwegen vermeiden helfen [39].

\section{Kortikosteroide}

Ob inhalative Kortikosteroide die entzündlichen Veränderungen des Atemwegsepithels und insbesondere das „epithelial shedding“ rückgängig machen können, ist nicht unumstritten. Laitinen et al. [5] demonstrierten zwar an histologischen Bildern des Atemwegsepithels eindrucksvoll den restaurierenden Effekt einer dreimonatigen Budesonid-Inhalationstherapie, doch andere Autoren konnten diesen Effekt nicht reproduzieren und beobachteten ganz im Gegenteil z. B. eine Zunahme der Apoptose epithelialer Zellen im Tieroder im In-vitro-Experiment $[40,41]$. Die meisten Studien beobachteten aber unter der Langzeittherapie mit inhalativen Kortikosteroiden eine Reduktion der epithelialen Entzündung, einen Anstieg der zilientragenden Zellen und eine Reduktion der Goblet-Zellmetablasie, der Drüsenzellzahl mit konsekutiver Mukusreduktion [42]. 
Kortikosteroide können nur partiell Remodelingprozesse rückgängig machen.

Auch in Bezug auf die Reduktion der Basalmembrandicke durch eine inhalative Kortikosteroidtherapie gibt es Studien, die einen positiven, und welche, die keinen Effekt fanden. Selbst wenn ein solcher Erfolg Z.B. durch eine hochdosierte und lange Therapie erzielbar ist und eine Reduktion der Kollagen-Typ-III-Deposition und Anstieg der Gewebe-Metalloprotease-1-Inhibitoren untermauert werden konnte, erscheint unklar, ob der beobachtete Effekt einen direkten Einfluss auf eine verbesserte Lungenfunktion besitzt $[14,42]$. Kortikosteroide reduzieren Interleukine in den Atemwegen, nicht jedoch TGF-ß, Gewebe-Proteoglykane oder Kollagen, was darauf hinweist, dass sie nur bestimmte Faktoren der Lamina propria beeinflussen und manche im Rahmen der asthmatischen Entzündung entstandenen Veränderungen der Lamina propria allerdings nicht reversibel bzw. therapeutisch nicht beeinflussbar sind [43].

Inhalative Kortikosteroide reduzieren die Menge an kleinen Blutgefäßen und generell die Angiogenese in den Atemwegen. Diese Beobachtung korrelierte mit der Reduktion der Basalmembrandicke, der bronchialen Reaktivität und einer Verbesserung der FEV1. Dabei scheinen aber die verschiedenen inhalativen Präparate eine unterschiedliche Effektivität zu besitzen, da Fluticason und Budesonid effektiver als Beclomethason waren. Die beobachteten Remodeling inhibierenden Effekte scheinen auf der Reduktion von Wachstumsfaktoren, insbesondere dem VEGF zu beruhen $[44,45]$.

Kortikosteroide reduzieren am besten die Aktivität von Myozyten.

Den größten Anti-Remodelingeffekt haben Kortikosteroide auf die glatten Muskelzellen. Sie reduzieren nicht nur die proinflammatorischen, für die Muskelproliferation und -funktion bedeutsamen Chemokine und Zytokine, sondern stoppen in der G1-Phase des Zellzyklus und hemmen proliferativ wirkende Wachstumsfaktoren und Mitogene via G-Protein-coupled-Rezeptoren [46]. Ferner unterdrücken sie die Expression kontraktiler Proteine und sekretorischer Fähigkeiten von Myozyten. In letzteren verhindern sie z.B. die Transskription von GM-CSF (granulocyte macrophagecolony stimulating factor). Diese Effekte sind nicht ECM-abhängig, da Kortikosteroide offenbar keinen oder keinen wesentlichen Einfluss auf ECM-Proteine besitzen [47].

\section{Leukotrien-modifizierende Substanzen}

Pranlukast und Zafirlukast, beide sind allerdings in Deutschland nicht zugelassene Leukotrienrezeptorantagonisten, unterdrücken die im Tier- experiment durch Allergene induzierte Entzündung des Atemwegsepithels und deren hypersekretorische Komponente [48]. Leukotrienrezeptorantagonisten reduzieren ebenso wie inhalative Kortikosteroide den Blutfluss in der Atemwegsmukosa, die Angiogenese, Effekte, die auch auf einer Hemmung der VEGF-Gewebespiegel beruhen. $10 \mathrm{mg}$ Montelukast hatte diesbezüglich bei Asthmapatienten den gleichen Effekt wie $400 \mu \mathrm{g}$ Fluticason [45].

Bestimmte antientzündliche Effekte von Leukotrieninhibitoren entsprechen ungefähr denen von Kortikosteroiden.

Zudem reduzierte es die Muskelmasse der glatten Muskulatur in den Atemwegen und die subepitheliale Fibrose in Ovalbumin-sensibilisierten Mäusen [49].

\section{Tiotropiumbromid}

Tiotropiumbromid ist kein beim Asthma zugelassenes Medikament, obwohl zunehmend Studien publiziert werden, die auch eine Effektivität bei dieser Erkrankung nachweisen [50,51]. Im Tierexperiment und im In-vitro-Ansatz ist die antientzündliche Wirkung von Tiotropiumbromid nachgewiesen worden. Es verhindert die Verdickung und reduziert die Kontraktilität glatter Muskelzellen, es reduziert die Myosinexpression und hat antichemotaktische Eigenschaften [5256].

\section{Tiotropiumbromid wirkt nicht nur bronchodilatativ.}

Zudem inhibiert Tiotropium über die Rezeptorblockade von Muskarinrezeptoren die entzündungsbedingte Kollagensynthese von Fibroblasten $[57,58]$ und führt zu einer Senkung der LPS (Lipopolysaccarid)-induzierten Neutrophilie, Goblet-Zellmenge, Kollagendeposition und der Anzahl von Blutgefäßen in der glatten Atemwegsmuskulatur [53]. Allerdings hat es keinen inhibitorischen Effekt auf die Emphysementstehung im COPD-Meerschweinchenmodell [53]. In Ovalbumin-sensibilisierten Meerschweinchen war Tiotropiumbromid ebenso effektiv wie Budesonid in der kompletten Prävention der Hypertrophie von Bronchialschleimdrüsen, in einer partiellen Hemmung MUC5AC-positiver Goblet-Zellen und eosinophiler Infiltrate [59].

\section{TNF-Inhibition}

Obwohl wegen der vielen Nebenwirkungen und teilweise auch geringen Effektivität die Therapie mit einem TNF(Tumornekrosefaktor-alpha)- $\alpha$ Antikörper keine Therapieoption für das Asthma ist, so zeigen Studien mit Etanercept und Infliximab, dass beide Antikörper eine antientzündliche Wirkung besitzen. So ließen sich nicht nur die Exazerbationsrate, sondern auch die erhöhten Zytokinspiegel im Sputum senken [60-62].

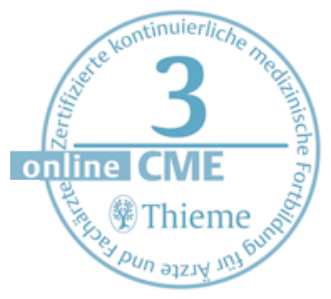


TNF- $\alpha$-Inhibition ist keine realistische Therapieoption, während die IgE-Hemmung für das schwere allergische Asthma zugelassen ist.

\section{IgE-Hemmung}

Omalizumab ist der einzige zur Therapie des schweren allergischen Asthmas zugelassene IgEAntikörper. Folgende klinische Effekte sind belegt: Verbesserung der FEV1, der Lebensqualität, Reduktion der für eine Asthmastabilisierung notwendigen systemischen Kortikosteroidmenge und Reduktion der rasch wirksamen beta-2-Mimetika-Bedarfstherapie [63-66]. Zudem führt Omalizumab zur Senkung diverser Entzündungsmarker einschließlich der bronchialen und peripheren Eosinophilenzellmenge, des GM-CSF und der Interleukine IL-2, IL-4, IL-5 und IL-13 sowie Endothelin im Atemkondensat [67]. In Ovalbumin-sensibilisierten Mäusen hatte intravenös appliziertes Omalizumab folgende Anti-Remodelingeffekte: Reduktion der eosinophilen Entzündung, der Interleukine IL-5 und IL-13, nicht jedoch IL-10, TGF-ß und Aktivin A [68].

\section{Zytokininhibition}

Ähnlich wie auch in der Onkologie, so gibt es auch beim Asthma und bei der COPD seit langem Bestrebungen, Entzündungswege zielgerichtet $\mathrm{zu}$ hemmen [69-71].

Zytokine sind das primäre Target eines zielgerichteten antientzündlichen Therapieansatzes.

Beispiele hierfür sind der Einsatz von Antikörpern gegen Interleukine oder Immunglobuline [63,70 -72]. Folgende Substanzen (Auswahl) wurden tierexperimentell auf ihre Fähigkeit evaluiert, Remodelingprozesse zu hemmen und sogar umzukehren:

- CCR3 (chemokine [C-C motif] receptor type 3)Antagonist [73]:

- Reduktion von Eosinophilen, Prävention von Goblet-Zellhyperplasie, subepitheliale Fibrose und Akkumulation von Myofibroblasten

- IL-4-Antikörper und IL-4-Rezeptor(R) $\alpha$ antisense Oligonucleodide [74-76]:

- Antiinflammatorischer Effekt, Reduktion Goblet-Zellen und Kollagendeposition

- IL-5-Antikörper [77, 78]:

- Eliminiert Blut- und Sputum- und reduziert um ca. die Hälfte Gewebe-Eosinophilie.

- Reduktion in Tenascin, Lumican und Prokollagen III im subepithelialen Kompartiment der Atemwege

- IL-13-Antikörper [69,79-81]:

- Reduktion von Eosinophilen, Mukusproduktion und Kollagendeposition

- TGF-ß-Antikörper und TGF-ß-Rezeptor-IKinase [82]:
- TGF-ß-Antikörper: Reduktion peribronchialer ECM-Deposition, Muskelzellproliferation und Mukusproduktion

- TGF-ß-Rezeptor-I-Kinase: Reduktion von Eosinophilen, CD2-positive T-Lymphozyten, zelluläre Smad2 /3 Expression, Epithelzellund Muskelzellproliferation sowie GobletZellhyperplasie

- Tryptase-Inhibitor [83]

- Reduktion von Eosinophilen, IL-4 und IL-13 in broncho-alveolärer Lavageflüssigkeit, Goblet-Zellhyperplasie, Mukusproduktion und peribronchiale Ödeme

- Oligodeoxynucleotide (CpG-DNA) [84]:

- Reduktion VEGF, TGF-B, CD4 T-Lymphozyten, Metalloproteinase-9, Kollagendeposition, Mukusproduktion, Angiogenese und Abbau schon peribronchial deponierten Kollagens

- Toll-like-Rezeptor 7/8 Ligand [85]:

- Reduktion der bronchialen Entzündung sowie Th1/Th2-Zytokine, Goblet-Zellhyperplasie. Anstieg der Muskelmasse via Proliferationssteigerung

\section{Phosphodiesteraseinhibitor}

Roflumilast ist ein selektiver Phosphodiesterase 4 (PDE-4)-Hemmer, der zur Therapie der COPD III und IV zugelassen ist. In humanen Muskelzellen der Atemwege reduzierte Roflumilast die peribronchiale Entzündung (Reduktion der eosinophilen Granulozyten), die Menge an subepithelialem Kollagen und die Dicke der Epithelschicht in den Atemwegen, nicht jedoch TGF- $ß$. In vitro reduzierte die Substanz die CTGF- und ECM-Deposition glatter Muskelzellen [86,87]. Diese Effekte waren mit denen von Dexamethason vergleichbar, wenngleich Dexamethason auch TGF- $\beta$ hemmte [86].

Roflumilast hemmt die peribronchiale Entzündung und wirkt antiproliferativ.

Die antiproliferative Eigenschaft von Roflumilast wurde eindrucksvoll in mit Bleomycin behandelten Mäusen demonstriert. In diesem Modell verminderte diese Substanz das Bleomycin-induzierte pulmonale Hypdroxyprolin, das Ausmaß der medikamentös ausgelösten Lungenfibrose, der rechts-ventrikulären Hypertrophie, der Muskelstärke intraazinärer Lungengefäße. Diese Effekte verliefen parallel zur Reduktion von TNF- $\alpha$, TGF$\beta$, Gewebewachstumsfaktoren, $\alpha(I)-K o l l a g e n$, Endothelin-1 und Mucin (Muc5ac) im Lungengewebe und teilweise auch in der bronchoalveolären Lavage, in der auch IL-13 im Vergleich zu den Roflumilast-negativen, mit Bleomycin behandelten Tieren verringert war. In diesem Modell waren die antifibrotischen Effekte von Roflumilast stärker als die von Methylprednisolon [88]. 


\section{Statine}

\section{$\nabla$}

Verschiedene Studien testeten, ob Statine zusätzliche Effekte als nur die Cholesterinsenkung besitzen. Klinische Studien zeigten beim Asthma, unabhängig davon, ob sie statt inhalativer Kortikosteroide oder zusammen mit ihnen gegeben wurden, klinisch keine bessere Wirkung als Plazebo. Allerdings besitzen sie pleiotrope, d.h. antientzündliche sowie antifibrotische Effekte auf die Endothelzellenfunktion, womit das Remodeling von Gefäßen, glatten Muskelzellen und Zellen der Atemwege inhibiert wird [89]. So hemmen Statine HMG-CoA (3-hydroxy-3-Methylgutaryl-Coenzym A)-Reduktase, in dessen Folge das daraus entstehende Mevalonat reduziert wird. Mevalonat stimuliert die TGFß1-vermittelte Fibronektinexpression über die Isoprenoidsynthese. Schaafsma et al. zeigten an ex vivo kultivierten und von Asthma- und Nicht-Asthmapatienten isolierten Fibroblasten, dass Simvastatin über die Regulation von Geranylgeranyltransferase-1 TGFß1 reduziert und die Fibroblastenaktivität hemmt [90].

Statine sind auch beim Asthma mehr als nur Cholesterinhemmer.

Ein ähnlicher Effekt ist mit Simvastatin bzw. Lovastatin an glatten Atemwegsmuskeln, die von sensibilisierten Mäusen oder Patienten mit einem Asthma isoliert wurden, beobachtet worden [90, 91]. Simvastatin hemmt die Goblet-Zellhyperplasie in Ovalbumin-sensibilisierten Mäusen und verstärkt die antientzündliche Wirkung von inhalativen Kortikosteroiden via Induktion der Indoleamin-2, 3-Dioxygenase [92, 93].

\section{Andere therapeutische Optionen}

Eine Fülle anderer proentzündlich wirkender Faktoren, die beim Asthma Remodelingprozesse auslösen, unterhalten und steuern, sind in den letzten Jahren identifiziert worden, die sich theoretisch alle für eine therapeutische Intervention eignen würden. Neben den hier besprochenen Substanzen werden und wurden in klinischen Studien untersucht: Endothelin-1-Rezeptorantagonisten, PDGF-Rezeptor-Kinaseinhibitoren, Kalziumkanalblocker, FLAP-Inhibitoren, Enzymblocker (Antiproteasen, Tryptaseinhibitoren) u.v.a.m. Gentherapeutisch wird versucht, die Transskription und Translation proentzündlicher Mediatoren durch Antisense-Oligonukleotiden, siRNA oder Lentivirus-vermittelte RNA-Interferenz zu unterbinden. Obwohl diese Ansätze ausschließlich zellbiologische in vitro oder tierexperimenteller Natur sind, weisen sie auf die große Bedeutung hin, die eine Hemmung des Remodeling aus therapeutischer Sicht haben könnte.
Viele andere antientzündlich wirkende Substanzen befinden sich in der Entwicklung.

\section{Thermoplastie}

$\checkmark$

Bei diesem Verfahren wird bronchoskopisch Wärme $\left(65^{\circ} \mathrm{C}\right)$ im Bereich einiger subsegmentaler Bronchienabschnitte appliziert. Die hypertrophierte glatte Muskulatur bildet sich in diesen Bereichen histologisch nachweisbar zurück, was zu einer leichten FEV1- und Lebensqualitätsverbesserung bei gleichzeitiger Reduktion der bronchialen Hyperreaktivität und der Exazerbationsrate führt $[20,21]$. Allerdings ist der Mechanismus, wie die letztendlich punktuelle Wärmeapplikation zu diesen positiven Effekten führt, wenig verstanden. Somit ist gegenwärtig auch unklar, welcher Asthma-Phänotyp besonders von dieser Therapieform einen Nutzen zieht \{Wahidi, 2012 9735/id\}.

\section{Fazit}

Die asthmatische Entzündung in den Atemwegen ist komplex und führt zu strukturellen Veränderungen in den Atemwegen, die reversibel oder aber irreversibel sein können. Die Wahrscheinlichkeit für letzteres steigt mit der Dauer, der Intensität und der fehlenden oder unzureichenden antiinflammatorischen Therapie. Obwohl viele Substanzen in der Lage sind, das Remodeling auf sehr unterschiedliche Art zu hemmen, besitzen sie nicht notwendigerweise auch eine gute klinische Wirksamkeit. Umgekehrt hemmt auch die derzeit uns zur Verfügung stehende Substanzgruppe mit der stärksten und breitesten antiinflammatorischen Wirksamkeit, nämlich die Kortikosteroide, keineswegs alle Remodeling-relevanten Strukturveränderungen. Ungeachtet dessen sind Kortikosteroide äußerst effektiv und bilden daher das wichtigste therapeutische Standbein in der Asthmatherapie. Wie in dieser Übersicht gezeigt wurde, gibt es viele Substanzen und noch viel mehr theoretisch mögliche Therapieansätze, die in vitro, tierexperimentell und auch am Menschen diese Strukturveränderungen hemmen bzw. die sich für einen antientzündlich wirkenden Behandlungsansatz eignen würden. Letztendlich ist aber für den Patienten nur der klinische Erfolg von Bedeutung, d.h. die Übersetzung des zellbiologischen Effekts auf die Verbesserung klinischer Parameter. Dieser Transfer gelingt gegenwärtig allerdings nur wenigen Substanzen, obwohl die berechtigte Hoffnung bleibt, dass sich in Zukunft das therapeutische Spektrum klinisch bedeutsamer antientzündlicher Wirkstoffe erweitert.

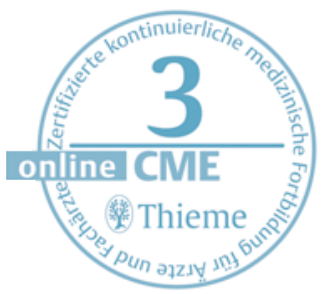




\section{Interessenkonflikt}

A. Gillissen: Referententätigkeit und Reisekostenunterstützung von Nycomed, Novartis; Referententätigkeit, Reisekostenunterstützung und Forschungsunterstützung von Boehringer Ingelheim.

\section{Literatur}

1 Elias JA, Zhu Z, Chupp G et al. Airway remodeling in asthma. Proc Am Thorac Soc 2009; 6: 301-305

2 Girodet PO, Ozier A, Bara I et al. Airway remodeling in asthma: new mechanisms and potential for pharmacological intervention. Pharmacol Ther 2011; 130: 325-337

3 Jeffery $P K$. Structural and inflammatory changes in COPD: a comparison with asthma. Thorax 1998; 53: $129-136$

4 Jeffery PK. Remodelling and inflammation of bronchi in asthma and chronic obstructive pulmonary disease. Proc Am Thorac Soc 2004; 1: 176- 183

5 Laitinen LA, Laitinen A, Haahtela T. A comparative study of the effects of an inhaled corticosteroid, budesonide, and a beta 2-agonist, terbutaline, on airway inflammation in newly diagnosed asthma: a randomized, doubleblind, parallel-group controlled trial. J Allergy Clin Immunol 1992; 90: 32-42

6 Ordonez C, Ferrando R, Hyde DM et al. Epithelial desquamation in asthma: artifact or pathology ? Am J Respir Crit Care Med 2010; 162: 2324-2329

7 Holgate ST, Roberts G, Arshad HS et al. The role of the airway epithelium and its interaction with environmental factors in asthma pathogenesis. Proc Am Thorac Soc 2009; 6: 655-659

8 Takeyama K, Fahy JV, Nadel JA. Relationship of epidermal growth factors to goblet cell production in human bronchi. Am J Respir Crit Care Med 2001; 163: 511 - 516

9 Shim JJ, Dabbagh K, Ueki IF et al. IL-13 induced mucin production by stimulation epidermal growth factor receptors and by activating neutrophils. AmJ Physiol Lung Cell Mol Physiol 2001; 280: L134-L140

10 Holgate ST. The airway epithelium is central to the pathogenesis of asthma. Allergol Int 2008; 57: 1-10

11 Payne DN, Rogers AV, Adelroth E et al. Early thickening of the reticular basement membrane in children with difficult asthma. Am J Respir Crit Care Med 2003; 167: 78 82

12 Bergeron C, Tulic MK, Hamid Q. Airway remodelling in asthma: from benchside to clinicial practice. Can Respir J 2010; 17: e85-e94

13 Yick CY, von der Thüsen JH, Bel EH et al. In vivo imaging of the airway wall in asthma: fibered confocal fluorescence microscopy in relation to histology and lung function. Respir Res 2011; 12: 85

14 Milanese M, Crimi E, Scordamaglia A et al. On the functional consequences of bronchial basement membrane thickening. J Appl Physiol 2001; 91: 1035 - 1040

15 Palmans E, Kips JC, Pauwels RA. Prolonged allergen exposure induces structural airway changes in sensitized rats. Am.J.Respir.Crit.Care Med 2000; 161: 627-635

16 Westergren-Thorsson G, Chakir J, Lafreniere-Allard MJ et al. Correlation between airway responsiveness and proteoglycan production by bronchial fibroblasts from normal and asthmatic subjects. Int J Biochem Cell Biol 2002; 34: $1256-1267$

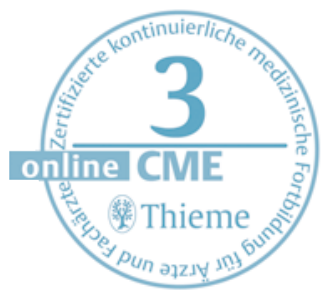

17 Suzuki R, Kato T, Miyazaki Y et al. Matrix metalloproteinases and tissue inhitibors of matrix metalloproteinases in sputum from patients with bronchial asthma. J Asthma 2001; 38: 477 - 484

$18 \mathrm{Ma}$ X, Cheng Z, Kong $\mathrm{H}$ et al. Changes in biophysical and biochemical properties of single bronchial smooth muscle cells from asthmatic subjects. Am J Physiol (Lung Cell Mol Physiol) 2002; 283: L1181 - L1189
19 Stephens NL, Fust A, jiang $H$ et al. Isotonic relaxation of controll and sensitized airway smooth muscle. Can J Physiol Pharmacol 2005; 83: 941 - 951

20 Cox G, Thomson NC, Rubin AS et al. Asthma control during the year after bronchial thermoplasty. N Engl J Med 2007; 356: $1327-1337$

21 Cox G, Miller D, McWilliams A et al. Bronchial thermoplasty for asthma. Am J Respir Crit Care Med 2006; 173: 965-969

22 Murphy DM, O'Byrne PM. Recent advances in the pathophysiology of asthma. Chest 2010; 137: 1417-1426

23 Koziol-White C], Panettieri RA Jr. Airway smooth muscle and immunomodulation in acute exacerbations of airway disease. Immunol Rev 2011; 242: 178 - 185

24 Johnson PR, Roth M, Tamm M et al. Airway smooth muscle cell proliferation is increased in asthma. Am J Physiol (Lung Cell Mol Physiol) 2001; 164: 474-477

25 Hollins F, Kaur D, Yang $W$ et al. Human airway smooth muscle promotes human lung mast cell survival proliferation, and constitutive activation: cooperative roles for CADM1, stemm cell factor, and IL-6. J Immunol 2008; 181: $2772-2780$

26 James AL, Bai TR, Mauad T et al. Airway smooth muscle thickness in asthma is related to severity but not duration of asthma. Eur Respir ] 2009; 34: 1040 - 1045

27 Grainge CL, Lauri CK, Ward JA et al. Effect of bronchoconstriction on airway remodeling in asthma. $\mathrm{N}$ Engl J Med 2011; 364: 2006 - 2015

28 Tschumperlin DJ, Drazen JM. Mechanical stimuli to airway remodeling. Am J Respir Crit Care Med 2001; 164: 90 S94

29 Black JL, Roth M, Lee J et al. Mechanisms of airway remodeling. Am J Respir Crit Care Med 2001; 164: S66

30 Yan ZQ, Hansson GK, Skoogh BE et al. Induction of nitric oxide synthase in a model of allergic occupational asthma. Allergy 1995; 50: 760-764

31 Williams MC. Maximizing morphologic data from lung biopsies from normal and asthmatic humans. Am J Respir Crit Care Med 1994; 150: 2 - 5

32 Gosens $R$, Zaagsma J, Meurs $H$ et al. Muscarinic receptor signaling in the pathophysiology of asthma and COPD. Resp Research 2007; 7: DOI 10.1186/1465-9921-7-73

33 Gosens R, Zaagsma J, Bromhaar MG et al. Acetylcholine: a novel regulator of airway smooth muscle remodelling? Eur J Pharmacol 2005; 500: 193-201

34 Slats AM, Janssen LI, Schadewijk vA et al. Expression of smooth muscle and extracellular matrix proteins in relation to airway function in asthma. J Allergy Clin Immunol 2008; 121: $1196-1292$

35 Dekkers BG, Schaafsma D, Nelemans SA et al. Extracellular matrix proteins differentially regulate airway smooth muscle phenotype and function. Am J Physiol Lung Cell Mol Physiol 2007; 292: L1405-L1413

36 Gillissen A, Richter A, Oster $\mathrm{H}$ et al. Efficacy and safety of once or twice daily inhalation of extrafine HFA beclomethasone dipropionate in patients with mild to moderate asthma. J Physiol Pharmacol 2007; 58: 233 - 241

37 Buhl R, Berdel D, Criée C-P et al. Leitlinie zur Diagnostik und Therapie von Patienten mit Asthma der Deutschen Atemwegsliga und der Deutschen Gesellschaft für Pneumologie und Beatmungsmedizin e.V. Pneumologie 2006; 60: 139-183

38 GINA Executive and Science Committee (Hrsg). Global Initiative for Asthma (GINA). Global strategy for asthma management and prevention Bethesda, USA: U.S. Department of Health and Human Services; 2010

39 Currani SR, Viswanathan RK, Busse WW. What effect does asthma treatment have on airway remodeling? Current perspectives J Allergy Clin Immunol 2011; 128: 439448

40 Erjefalt JS, Erjefalt I, Sundler $F$ et al. In vivo restitution of airway epithelium. Cell Tissue Res 1995; 281: 305 - 316

41 Dorscheid DR, Wojcik KP, Sun S et al. Apoptosis of airway epithelial cells induced by corticosteroids. Am J Respir Crit Care Med 2001; 164: 1939-1947 
42 Hoshino M, Takahashi M, Takai Y et al. Inhaled corticosteroids decrease subepithelial collagen deposition by modulation of the balance between matrix metalloproteinase- 9 and tissue inhibitor of metalloproteinas-1 expression in asthma. J Allergy Clin Immunol 1999; 104: 356 363

43 Mauad T, Bel EH, Sterk PJ. Asthma therapy and airway remodeling. J Allergy Clin Immunol 2011; 120: 997 - 1009

44 Felits $B N$, Wignarajah $D$, Reid DW et al. Effects of inhaled fluticasone on angiogenesis and vascular endothelial growth factor in asthma. Thorax 2007; 62: 314-319

45 Mendes ES, Pereira A, Danta l et al. Comparative bronchial vasoconstrictive efficacy of inhaled glucocorticosteroids. Eur Respir J 2003; 21: 989 -993

46 Vlahos R, Lee KS, Guida E et al. Differential inhibition of thrombin- and EGF-stimulated human cultured airway smooth muscle proliferation by glucocorticoids. Pulm Phamacol Therapeutics 2003; 16: $171-180$

47 De Kluijver J, Schrumpf JA, Evertse CE et al. Bronchial matrix and inflammation respond to inhaled steroids despite ongoing allergen exposure in asthma. Clin Exp Allergy 2005; 35: 1361 - 1369

48 Muz MH, Deveci F, Bulut $Y$ et al. The effects of low dose leukotriene receptor antagonist therapy on airway remodeling und cystenyl leukotriene expression in a mouse asthma model. Exp Mol Med 2006; 38: 109-118

49 Henderson WR Jr, Chiang GK, Tien YT et al. Reversal of allergen-induced airway remodeling by CysLT1 receptor blockade. Am J Respir Crit Care Med 2006; 173: 718 728

50 Peters SP, Kunselman S, Icitovic $N$ et al. Tiotropium bromide step-up therapy for adults with uncontrolled asthma. N Engl J Med 2010; 363: 1715-1726

51 O'Connor BJ, Towse LJ, Barnes PJ. Prolonged effect of tiotropium bromide on methacholine-induced bronchoconstriction in asthma. Am J Respir Crit Care Med 1996; 154: $876-880$

52 Vacca G, Randerath W, Gillissen A. Inhibition of granulocyte migration by tiotropium bromide. Respir Res 2011; 12: 24

53 Pera T, Zuidhof A, Valadas / et al. Tiotropium inhibits pulmonary inflammation and remodelling in guinea pis model of COPD. Eur Respir ] 2011; 38: 789-796

54 Pieper MP, Chaudhary NI, Park JE. Acetylcholine-induced proliferation of fibroblasts and myofibroblasts in vitro is inhibited by tiotropium bromide. Life Sci 2007; 80 2270- 2273

55 Bühling F, Lieder N, Kühlmann UC et al. Tiotropium suppresses acetylcholine-induced release of chemotactic mediators in vitro. Respir Med 2007; 101: 2386 - 2394

56 Gosens R, Bos ST, Zaagsma J et al. Protective effects of tiotropium bromide in the progresse of airway smooth muscle remodeling. Am J Respir Crit Care Med 2005; 171: 1096 - 1102

57 Matthiesen S, Bahulayan A, Kempkens S et al. Muscarinic receptors mediate stimulation of human lung fibroblast proliferation. Am J Respir Cell Mol Biol 2006; 35: 621 627

58 Haag S, Matthiesen S, Juergens UR et al. Muscarinic receptors mediate stimulation of collagen synthesis in human lung fibroblasts. Eur Respir J 2008; 32: 555 - 562

59 Bos IS, Gosens R, Zuidhof $A B$ et al. Inhibition of allergeninduced airway remodelling by tiotropium and budesonide: a comparision. Eur Respir ] 2007; 30: 653-661

60 Howarth P, Babu KS, Arshad HS et al. Tumour necrosis factor (TNFalpha) as a novel therapeutic target in symptomatic corticosteroid dependent asthma. Thorax 2005; 60: $1012-1018$

61 Erin EM, Leaker BR, Nicholson GC et al. The effects of a monoclonal antibody directed against tumor necrosis factor-alpha in asthma. Am J Respir Crit Care Med 2006; 174: $753-762$

62 Berry MA, Hargadon B, Shelley $M$ et al. Evidence of a role of tumor necrosis factor alpha in refractory asthma. N Engl J Med 2006; 354: 697 -708
63 Buhl R, Hanf G, Solér M et al. The anti-lgE antibody omalizumab improves asthma-related quality of life in patients with allergic asthma. Eur Respir J 2002; 20: 1088- 1094

64 Fahy JV, Fleming $\mathrm{E}$, Wong $\mathrm{HH}$ et al. The effect of an anti$\mathrm{lgE}$ monoclonal antibody on the early- and late phase responses to allergen inhalation in asthmatic subjects. Am J Respir Crit Care Med 1997; 155: 1828 - 1834

65 Lanier BQ, Corren J, Lumry W et al. Omalizumab is effective in the long-term control of severe all asthma. Ann Allergy Asthma Immunol 2003; 91: 154 - 159

66 Solér M, Matz J, Townley $R$ et al. The anti-lgE antibody omalizumab reduces exacerbations and steroid requirement in allergic asthmatics. Eur Respir ] 2001; 18: 254 261

67 Rabe KF, Calhoun WJ, Smith $N$ et al. Can anti-lgE therapy prevent airway remodeling in allergic asthma? Allergy 2011; 66: $1142-1151$

68 Kang JY, Kim JW, Kim SJ et al. Inhibitory effects of anti-immunoglobulin $\mathrm{E}$ antibodies on airway remodeling in a murin model of chronic asthma. J Asthma 2010; 47: $374-380$

69 Krause S, Behrends J, Borowski A et al. Blockade of interleukin-13-mediated cell activation by a novel inhibitory antibody to human IL-13 receptor alpha1. Mol Immunol 2006; 43: 1799-1807

70 Myrtek D, Knoll M, Matthiesen T et al. Expression of interleukin-13 receptor alpha 1-subunit ond peripheral blood eosinophils is regulated by cytokines. Immunology 2004; 112: $597-604$

71 Julius $P$, Hochheim D, Boser $K$ et al. Interleukin-5 receptors on human lung eosinophiles after segmental allergen challenge. Clin Exp Allergy 2004; 34: 1064-1070

72 Bundschuh DS, Eltze M, Barsig J et al. In vivo efficacy in airway disease models of roflumilast, a novel orally active PDE4 inhibitor. J Pharmacol Exp Ther 2001; 297: 280 289

73 Wegmann M, Göggel R, Sel S et al. Effects of a low-molecular-weight CCR-3 antagonist on chronic experimental asthma. Am J Respir Crit Care Med 2007; 36: 61 - 67

74 Hoshino M, Fujita Y, Saji J et al. Effect of suplatast tosilate on goblet cell metaplasie in patients with asthma. Allergy 2005; 60: $1394-1400$

75 Karras JG, Crosby JR, Guha M et al. Anti-inflammatory activity of inhaled IL-4 receptor-alpha antisense oligonucleotide in mice. Am J Respir Crit Care Med 2007; 36: $276-$ 285

76 Tomkinson A, Duez C, Cieslewicz G et al. A murine IL-4 receptor antagonist that inhibits IL-4- and IL-13-induced responses prevents antigen-induced airway eosinophilia and airway hyperresponsiveness. J Immunol 2001; 166: $5792-5800$

77 Shardonofsky F, Venzor J, Barrios $R$ et al. Therapeutic efficacy of an anti-IL-5 monocolonal antibody delivered into the respiratory tract in a murine model of asthma. J Allergy Clin Immunol 1999; 104: 215-221

78 Leckie MJ. Anti-IL-5 monoclonal antibodies: preclinical and clinical evidence in asthma models. Am J Respir Med 2003; 3: 245-259

79 Hashimoto T, Akiyama K, Kawaguchi H et al. Correlation of allergen-induced IL-5 and IL-13 production by peripheral blood T cells of asthma patients. Int Arch Allergy Appl Immunol 2004; 134: 7-11

80 Grunig G, Warnock M, Wakil AE et al. Requirement for IL-13 independently of IL-4 in experimental asthma. Science 1998; 282: $2261-2263$

81 Yang G, Vok A, Petley T et al. Anti-IL-13 monoclonal antibody inhibits airway hyperresponsiveness, inflammation and airway remodeling. Cytokine 2004; 28: 224-232

82 Fattouh R, Midence NG, Arias K et al. Transforming growth factor-beta regulates house dust mite-induced allergic airway inflammation but not airway remodeling. Am Respir Crit Care Med 2008; 177: 593-603

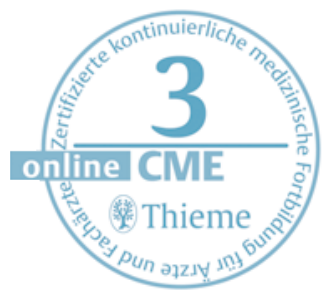


83 Oh SW, Pae CL, Lee DK et al. Tryptase inhibition blocks airway inflammation in a mouse asthma model. J Immunol 2002; 168: 1992 - 2000

84 Fanucchi MV, Schelegle ES, Baker GL et al. Immunostimulatory oligonucleotides attenuate airways remodelling in allergic monkeys. Am J Respir Crit Care Med 2004; 170: $1153-1157$

85 Camateros P, Tamaoka M, Hassan M et al. Chronic asthma-induced airway remodeling is prevented by Toll-like receptor-7/8 ligand S28463. Am J Respir Crit Care Med 2007; 175: 1241 - 1249

86 Kumar RK, Herbert C, Thomas PS et al. Inhitibtion of inflammatory and remodeling by roflumilast and dexamethasone in murine chronic asthma. J Pharmacol Exp Ther 2003; 307: 349-355

87 Burgess JK, Oliver BG, Poniris MH et al. A phosphodiesterase 4 inhibitor inhibits matrix protein deposition in airways in vitro. J Allergy Clin Immunol 2006; 118: 649657

88 Cortijo J, Iranzo A, Milara X et al. Roflumilast, a phosphodiesterase 4 inhibitor, alleviates bleomycin-induced lung injury. $\mathrm{Br}$ J Pharmacol 2009; 156: $534-544$
89 Camoretti-Mercado B. Targeting the airway smooth muscle for asthma treatment. Transl Res 2009; 154: 165 174

90 Schaafsma $D$, McNeill KD, Mutawe MM et al. Simvastatin inhibits TGFß1-induced fibronectin in human airway fibroblasts. Resp Research 2011; 12: 113 (e-pub)

91 Chiba Y, Sato S, Misawa M. Upregulation of geranylgeranyltransferase I in bronchial smooth muscle of mouse experimental asthma: its inhibition by lovastatin. J Smooth Muscle Res 2010; 46: 57-64

92 Maneechotesuwan K, Ekjiratrakul W, Kasetsinsombat $K$ et al. Statins enhance the anti-inflammatory effects of inhaled corticosteroids in asthma patients through increased induction of indoleamine 2, 3-dioxygenase. J Allergy Clin Immunol 2010; 126: 754-762

93 Zeki AA, Bratt JM, Rabowsky M et al. Simvastatin inhibits goblet cell hyperplasia and lung arginase in a mouse model of allergic asthma: a novel treatment for airway remodeling? Transl Res 2010; 156: 335-349

94 Wahidi MM, Kraft M. Bronchial thermoplasty for severe asthma. Am J Respir Crit Care Med 2011; 185: 709 - 714

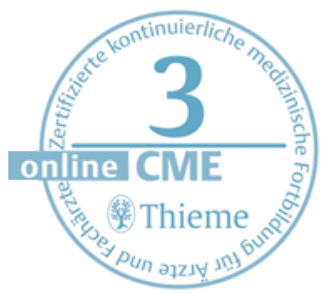




\section{CME-Fragen Remodeling beim Asthma}

1 Der Begriff „Remodeling“ ist nicht einheitlich definiert. Neben entzündlichen Veränderungen umfasst er:

A das Cor pulmonale

B strukturelle Veränderungen in den Atemwegen

C die Alveolitis

D die Epiglottitis

E extrapulmonale Erkrankung mit Auswirkungen auf die Lunge

2 Beim Asthma ist das Atemwegsepithel in folgender Weise mit betroffen:

A Steigerung zilientragender Zellen

B Verlust von Goblet-Zellen mit Versiegen der

Schleimproduktion

C Angiogenese

D Ein Epithelschaden senkt die bronchiale Empfindlichkeit.

E Steigerung der sekretorischen Funktion

3 Die extrazelluläre Matrix (ECM)

A garantiert den dreidimensionalen Aufbau von Nervenzellen.

B spielt in der asthmatischen Entzündung keine Rolle.

C wird beim Remodeling im Bereich der Lamina reticularis verändert, die fibrosiert.

D Entzündung der ECM bedingt eine Verdickung der Bronchialepithelschicht.

E Entzündung der ECM führt zu einer Erschlaffung der Atemwege.

\section{4}

\section{Die Bronchialmuskulatur}

A exprimiert bei der asthmatischen Entzündung vermehrt kontraktile Proteine.

B hypertrophiert bei der Entzündung und deren Muskelzellzahl nimmt ab.

C Hypertrophierte Muskelzellen relaxieren schneller als normale Muskelzellen.

D Die Muskelkraft ist disproportional zur Muskelmasse, was auch für die glatte Muskulatur gilt.

E Der Muskelphänotyp verändert sich beim Remodeling nicht.

A sind bei jungen Kindern mit Asthma noch nicht hypertrophiert.

B interagieren mit Mastzellen, indem diese die Proliferationsfähigkeit von Muskelzellen steigern.

C kommunizieren mit Bestandteilen der extrazellulären Matrix (ECM) und umgekehrt.

D sind beim Asthmapatienten für die Entwicklung der bronchialen Hyperreagibilität bedeutungslos.

E interagieren mit dem sympathischen Nervensystem.

\section{Kortikosteroide}

A haben den größten Anti-Remodelingeffekt auf die glatten Muskelzellen der Atemwege.

B verhindern und verbessern alle strukturellen Remodelingveränderungen in der Lamina propria bzw. in der ECM.

C haben keinen Einfluss auf die Angiogenese in den Atemwegen.

D steigern die kontraktilen Proteine von Myozyten.

E wirken alle gleich antiinflammatorisch.

7 Tiotropium könnte eine für das Asthma interessante Substanz sein, weil es

A keinen antientzündlichen Effekt besitzt und nur bronchodilatativ wirkt.

B chemotaktische Eigenschaften besitzt.

C die Emphysementstehung blockiert.

D die Verdickung von Muskelzellen und deren Kontraktilität verhindert.

E über ß2-Rezeptoren die Kollagensynthese von Fibroblasten stoppt.

8 Welche Antwort zu weiteren antientzündlichen Therapieformen beim Asthma stimmt?

A Omalizumab inhibiert das Immunglobulin G.

B Der Tumornekrosefaktor-alpha wird gut vertragen und senkt die Blut-Granulozyten.

C Die IL-4-Inhibition wirkt proinflammatorisch.

D Die meisten dieser Therapieoptionen kamen über das In-vitro-Experimentalstadium nicht hinaus.

E Die IL-5-Inhibition senkt Blut und Sputumeosinophilenanzahl.

Phosphodiesterase-4-Inhibitor

A ist für das Asthma III und IV zugelassen.

B ist wie Theophyllin ein unselektiver Hemmer der Phosphodiesterase.

C besitzt antientzündliche Effekte, die in vitro denen von Dexamethason bzw. Methylprednisolon ähneln.

D Roflumilast wirkt nur peribronchial, nicht aber im Lungenparenchym (nicht antifibrogen).

E hat proliferative Eigenschaften.

Statine, die neue Wundersubstanzgruppe,

A wirken breit antientzündlich, antifibrotisch in Gefäßen und sogar in Atemwegen.

B wirken über eine Steigerung der HMG-CoA-Reduktaseaktivität, womit die Entzündung gehemmt wird.

C bewiesen eine gute klinische Wirksamkeit beim Asthma.

D steigern über Mevalonat die TGFß1-vermittelte Fibronektinexpression.

E steigern die Aktivität glatter Muskelzellen, wie bei Simvastatin bzw. Lovastatin gezeigt wurde. 